Photogrammetric Record, xx(xxx): 000-000 (Month 20\#\#)

\title{
GEOMETRIC MODELLING AND CALIBRATION OF A SPHERICAL CAMERA IMAGING SYSTEM
}

\author{
DEREK D. LICHTI* (ddlichti@ucalgary.ca) \\ DAVID JARRON (dmjarron@ucalgary.ca) \\ WYNAND TREDOUX (wynand.tredoux@ucalgary.ca) \\ MOZHDEH SHAHBAZI (Mozhdeh.shahbazi@ucalgary.ca) \\ University of Calgary, Calgary, Canada \\ ROBERT RADOVANOVIC (rradovanovic@mcelhanney.net) \\ McElhanney Ltd., Calgary, Canada \\ * Corresponding author
}

\begin{abstract}
The Ladybug5 is an integrated, multi-camera system that features a near spherical field of view. It is commonly deployed on mobile mapping systems to collect imagery for $3 D$ reality capture. This paper describes an approach for the geometric modelling and self-calibration of this system. The collinearity equations of the pinhole camera model are augmented with five radial lens distortion terms to correct the severe barrel distortion. Weighted relative orientation stability constraints are added to the self-calibrating bundle adjustment solution to enforce the angular and positional stability between the Ladybug5's six cameras. Results are presented from two calibration datasets and an independent dataset for accuracy assessment. It is demonstrated that centimetre-level $3 D$ reconstruction accuracy can be achieved with the proposed approach. Moreover, the effectiveness of the lens distortion modelling is demonstrated. Image-space precision and object-space accuracy are improved by 92\% and 93\%, respectively, relative to a two-term model. The high correlations between lens distortion coefficients were not found to be detrimental to the solution. The mechanical stability of the system was assessed by comparing calibrations taken before and after ten months of routine camera system use. The results suggest sub-pixel interior orientation stability and millimetre-level relative orientation stability. Analyses of accuracy and parameter correlation demonstrate that a slightly relaxed weighting strategy is preferred to tightly-enforced relative orientation stability constraints.
\end{abstract}

KEYWORDS: camera calibration, multi-camera system, wide-angle lenses, relative orientation stability, Ladybug5 


\section{INTRODUCTION}

STATE-OF-THE-ART mobile mapping systems (MMSs) comprise multiple sensors for mapping complex terrestrial environments. In addition to navigation sensors (GNSS receivers, inertial measurement units, etc.) for trajectory determination, they generally include multiple imaging sensors such as laser scanners and digital cameras. Whether the MMS is vehicle-borne or backpack based, the Ladybug imaging systems from FLIR Integrated Imaging Solutions are a common choice to facilitate panoramic imaging in support of 3D reality capture. These systems, in particular the Ladybug3 and Ladybug5, comprise six cameras integrated in a rigid housing to provide a nearly spherical field-ofview. In their review of urban applications and methods, Wang et al (2019) summarize eight MMS systems, of which five include Ladybug5 devices. Others report MMSs incorporating Ladybug cameras on robotic (Schneider and Förstner, 2013), backpack (Rau et al, 2016) and land vehicle (Cui et al, 2017) platforms.

Despite the seemingly widespread use of these camera systems, few detailed reports exist about their geometric calibration. Schneider and Förstner (2013) report on the selfcalibration of a Ladybug3 system. In particular, they comment on deviations of the relative orientation between cameras from the manufacturer's values. Rau et al (2016) describe the self-calibration of the Ladybug5. They mention the need for higher-order radial lens distortion terms and report large residual systematic errors. Bosch et al (2015) describe a ray-tracing approach for underwater use of a Ladybug3 system. Jarron et al (2019a) provide a brief, initial report on the self-calibration of the Ladybug5. Much more detailed treatment is given herein.

The purpose of this paper is to present a geometric modelling approach and calibration assessment of a multi-camera imaging system, specifically, the FLIR Ladybug5. The system in question is first described in detail in order to give proper context to the mathematical modelling. Next, the geometric models used for the system observation equations, constraints and lens distortions are described. Experiments to test the effectiveness of the modelling approach are then described. The results and analyses that follow comprise several quantitative assessments:

1. The quality of the interior orientation parameters;

2. The effectiveness of the radial lens distortion model;

3. The quality of the relative orientation parameters; and

4. The 3D measurement accuracy as a result of the modelling approach.

\section{CAMERA SYSTEM DESCRIPTION}

The Ladybug5 Spherical Camera Imaging System from FLIR Integrated Imaging Solutions comprises six cameras integrated in a rigid housing (Fig. 1). Each camera is a Sony ICX655 (2/3" CCD; $3.45 \mu \mathrm{m}$ pixel spacing; 2048 x 2448 pixel image size; global shutter) fitted with a $4.4 \mathrm{~mm}$ focal length lens. The system can output uncompressed imagery at $5 \mathrm{~Hz}$ or JPEG imagery at $10 \mathrm{~Hz}$.

The horizontal cameras are mounted with a roll angle of $90^{\circ}$ so that the long dimension of the sensor format is vertically oriented. As a result, the corresponding horizontal and vertical angular fields-of-view of each camera are approximately $77.5^{\circ}$ and $87.6^{\circ}$, respectively. The angular overlap between any two adjacent horizontal cameras is 
approximately $5.5^{\circ}$. Their perspective centres nominally lie on a horizontal circle with an approximate radius of $60 \mathrm{~mm}$. The sixth camera is oriented so that it looks vertically upwards. Its perspective center is nominally $75 \mathrm{~mm}$ above the plane containing those of the horizontal cameras. The overlap of the vertical camera with the horizontal cameras' fields-of-view varies due to the rectangular image format.

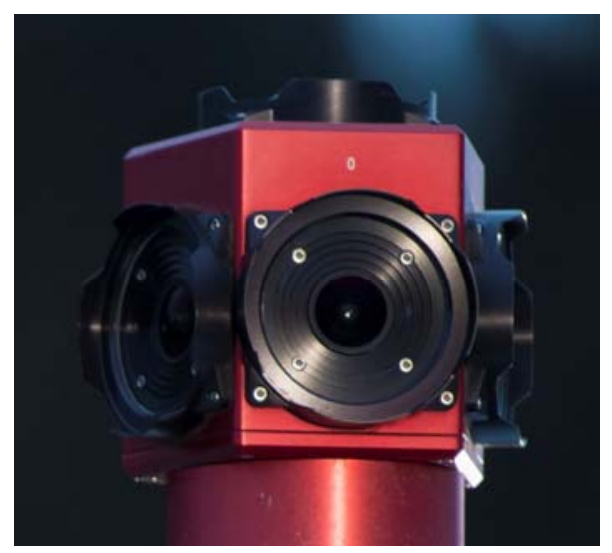

FIG. 1. The Ladybug5 system. (Image courtesy McElhanney Ltd).

METHODOLOGY

\section{Collinearity}

The imaging geometry of a multi-camera system comprising $n$ cameras is described by two sets of parameters: the $n$ sets of individual cameras' interior orientation parameters (IOPs); and the (n-1) sets relative orientation parameters (ROPs) between cameras. Observations in each individual camera are modelled by the collinearity condition that expresses the hypothesized straight-line relationship between object point $i(X, Y, Z)$, its image point $(x, y)$ and the perspective centre of image $j\left(X^{c}, Y^{c}, Z^{c}\right)$ :

$$
\begin{aligned}
& x_{i j}+\varepsilon_{x_{i j}}=x_{p_{j}}-c_{j} \frac{m_{11}\left(X_{i}-X_{j}^{c}\right)+m_{12}\left(Y_{i}-Y_{j}^{c}\right)+m_{13}\left(Z_{i}-Z_{j}^{c}\right)}{m_{31}\left(X_{i}-X_{j}^{c}\right)+m_{32}\left(Y_{i}-Y_{j}^{c}\right)+m_{33}\left(Z_{i}-Z_{j}^{c}\right)}+\Delta x_{i j} \\
& y_{i j}+\varepsilon_{y_{i j}}=y_{p_{j}}-c_{j} \frac{m_{21}\left(X_{i}-X_{j}^{c}\right)+m_{22}\left(Y_{i}-Y_{j}^{c}\right)+m_{23}\left(Z_{i}-Z_{j}^{c}\right)}{m_{31}\left(X_{i}-X_{j}^{c}\right)+m_{32}\left(Y_{i}-Y_{j}^{c}\right)+m_{33}\left(Z_{i}-Z_{j}^{c}\right)}+\Delta y_{i j}
\end{aligned}
$$

where $\left(x_{p}, y_{p}\right)$ are the coordinates of the principal point, $c$ is the principal distance, and $m_{p q}$ are elements of the rotation matrix $\mathbf{M}$, which is parameterized in terms of the sequential rotation angles $\omega, \phi$ and $\kappa$. 


\section{Lens Distortion Modelling}

The collinearity equations are augmented with both random error terms $\left(\varepsilon_{x}, \varepsilon_{y}\right)$ and correction terms $(\Delta x, \Delta y)$ that model the systematic errors that cause departures from collinearity due to lens distortion. The standard, two-term $\left(p_{1}, p_{2}\right)$ Brown-Conrady model is employed to model decentring distortion.

$$
\begin{aligned}
& \Delta x=p_{1}\left(r^{2}+2 \bar{x}^{2}\right)+2 p_{2} \overline{x y} \\
& \Delta y=p_{2}\left(r^{2}+2 \bar{y}^{2}\right)+2 p_{1} \overline{x y}
\end{aligned}
$$

where $r$ is radial distance with respect to the principal point and $(\bar{x}, \bar{y})$ are image coordinates reduced to the principal point.

The severe barrel distortion inherent to the wide-angle lenses of the Ladybug5 system requires five radial lens distortion terms $\left(k_{1}, k_{2}, k_{3}, k_{4}, k_{5}\right)$

$$
\begin{aligned}
& \Delta x=\bar{x}\left(k_{1} r^{2}+k_{2} r^{4}+k_{3} r^{6}+k_{4} r^{8}+k_{5} r^{10}\right) \\
& \Delta y=\bar{y}\left(k_{1} r^{2}+k_{2} r^{4}+k_{3} r^{6}+k_{4} r^{8}+k_{5} r^{10}\right)
\end{aligned}
$$

Though the use of more than three radial lens distortion coefficients is not commonly encountered, some software packages support inclusion of higher-order terms. Both the FLIR Ladybug5 SDK and Agisoft Photoscan (Agisolft LLC, 2018) support inclusion of the $k_{4}$ term while Photometrix CameraCalibrator supports the addition of both $k_{4}$ and $k_{5}$ (Photometrix, 2016).

Colour cameras can also possess chromatic aberrations due to light dispersion in the lens. It can have both longitudinal and transverse components. The former amounts to wavelength-dependent plane of best focus (principal distance) and the latter is wavelength-dependent radial lens distortion. An experiment to quantify the presence of chromatic aberration in the Ladybug5 system is reported in (Lichti et al, 2019c). No significant longitudinal chromatic effects were found and the lateral effect was only at the 1-2 pix level. Accordingly, these effects are omitted from the camera modelling herein.

\section{Model Identification}

A sequential model construction process is followed to identify significant terms in the radial lens distortion model. An initial self-calibrating bundle adjustment comprising a minimal set of IOPs is first performed. Generally this might only include the principal distance and the principal point and, perhaps, $k_{1}$. For the Ladybug5, it also included $k_{2}$ due to the very high barrel distortion. Statistical, graphical and information-theoretical analyses are then performed. The radial component of the image point residuals is plotted as a function of radial distance. If a systematic trend can be observed in the scatter plot, the distortion model is augmented by one term and the self-calibration adjustment is run again with the new model. The root mean square of the residuals, $r m s_{x y}$, (Remondino et al, 2017) is computed and analysed. 
If the added term is significant, then the $r m s_{x y}$ should be reduced by a significant amount. Judging the significance of the reduction to the $r m s_{x y}$ can be subjective and the parameter significance test ignores inherent parameter correlations. Therefore, some additional measures are computed. First, the statistical significance of each lens distortion parameter is examined using the univariate testing procedure described by Shortis et al (2001). Second, the Akaike Information Criterion (AIC; Akaike, 1974) is used to ascertain whether the new model is superior to the previous. If a systematic trend is still visible in the new set of residuals, then the model complexity is increased and the process repeated until all trends have been modelled. Accuracy assessment using independentlysurveyed check points can also be performed to measure the significance of the model improvement in object space.

\section{Relative Orientation Stability}

Several approaches exist for modelling the relative orientation multi-camera systems. An exhaustive review of all such methods is beyond the scope of this paper, so interested readers are referred to Detchev et al (2018). Broadly speaking, they can be summarized as belonging to one of two approaches:

1. Explicitly modelled relative orientation; and

2. Added constraints that enforce stability of the ROPs.

Each approach makes the assumption that the relative position and orientation of the cameras in their housing are mechanically stable.

In the first approach, one camera of the system is designated as the master camera and the remaining sensors are designated as slave cameras. The exterior orientation parameters (EOPs) of the master camera and the ROPs of the (n-1) cameras are estimated simultaneously. There is no need to derive the ROPs after the self-calibrating bundle adjustment since they explicitly appear in the model. Examples of this approach to modelling multi-camera systems can found for mobile mapping (Habib et al, 2011) and laboratory-based, structural deformation measurement (Detchev et al, 2018).

The second modelling approach includes $n$ sets of EOPs. The ROPs are not explicitly modelled. Instead, constraints are added to the multi-station bundle adjustment to express the assumed relative stability of the cameras. The actual ROPs are derived from the EOPs post-adjustment. Three position and three angular constraints can be written between each camera pair. A total of $6(n-1)$ independent constraint equations can be added to the self-calibration adjustment. This is the approach adopted for this work. It has been applied to airborne multi-camera sensors (Tommaselli et al, 2013), multi-camera fisheye lens systems (Campos et al, 2018), a general multi-projective system (Khoramshahi and Honkavaara, 2018), an underwater stereo camera system (Shortis, 2015) and a dual fluoroscopic imaging system (Lichti et al, 2015).

The base vector $\left(b_{X}, b_{Y}, b_{Z}\right)$ between cameras $i$ and $j$ can be defined as

$$
\left(\begin{array}{l}
b_{X} \\
b_{Y} \\
b_{Z}
\end{array}\right)_{i j}=\mathbf{M}_{i}\left(\begin{array}{c}
X_{j}^{c}-X_{i}^{c} \\
Y_{j}^{c}-Y_{i}^{c} \\
Z_{j}^{c}-Z_{i}^{c}
\end{array}\right)
$$


Note that the choice of image space in which the constraint is parameterized is arbitrary; camera has been used $i$ here. The stability of the base vector components between cameras $i$ and $j$ is enforced by constraining the difference in their base vector components at two camera locations ( 1 and 2 ) to be zero

$$
\mathbf{M}_{i_{1}}\left(\begin{array}{c}
X_{j}^{c}-X_{i}^{c} \\
Y_{j}^{c}-Y_{i}^{c} \\
Z_{j}^{c}-Z_{i}^{c}
\end{array}\right)_{1}-\mathbf{M}_{i_{2}}\left(\begin{array}{c}
X_{j}^{c}-X_{i}^{c} \\
Y_{j}^{c}-Y_{i}^{c} \\
Z_{j}^{c}-Z_{i}^{c}
\end{array}\right)_{2}=\left(\begin{array}{l}
0 \\
0 \\
0
\end{array}\right)+\left(\begin{array}{c}
\varepsilon_{\Delta b_{X}} \\
\varepsilon_{\Delta b_{Y}} \\
\varepsilon_{\Delta b_{Z}}
\end{array}\right)_{12}
$$

Others (Lerma et al, 2010; Tommaselli et al, 2013) report the option to constrain the base vector length rather than its three components.

The development of the angular ROP stability constraints requires the intermediate computation of the relative rotation matrix, which can be parameterized in terms of relative angles $\Delta \omega, \Delta \phi$ and $\Delta \kappa$.

$$
\Delta \mathbf{M}_{i j}=\mathbf{M}_{j} \mathbf{M}_{i}^{T}=\mathbf{R}_{3}\left(\Delta \kappa_{i j}\right) \mathbf{R}_{2}\left(\Delta \phi_{i j}\right) \mathbf{R}_{1}\left(\Delta \omega_{i j}\right)
$$

The constraints can be formulated in terms of the ROP angles themselves (Lichti et al, 2015) or as functions of the relative rotation matrix elements (King, 1995, Tommaselli et al, 2013). Here, the relative angles are constrained. Though the choice is perhaps somewhat subjective, the primary motivation is that the weighting (see below) for this approach is more intuitive because the constraint standard deviations can be expressed in angular units such as arc seconds rather than unit-less direction cosines. The relative angles can be derived from $\Delta \mathbf{M}_{i j}$ according to standard equations.

$$
\begin{aligned}
\Delta \omega_{i j} & =\arctan \left(\frac{-\Delta m_{32}}{\Delta m_{33}}\right) \\
\Delta \phi_{i j} & =\arcsin \left(\Delta m_{31}\right) \\
\Delta \kappa_{i j} & =\arctan \left(\frac{-\Delta m_{21}}{\Delta m_{11}}\right)
\end{aligned}
$$

It should be noted that an alternative angle parameterization is required to avoid the gimbal lock problem if the secondary relative rotation angle is $90^{\circ}$.

The angular stability constraints enforce the differences between the relative angles between cameras $i$ and $j$ captured at locations 1 and 2 to be zero. 


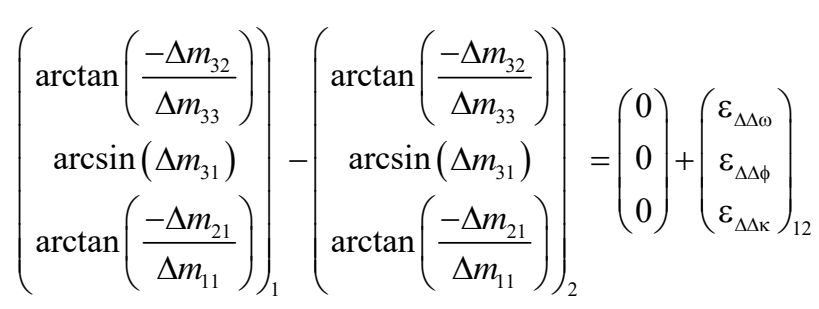

The ROP stability conditions can be incorporated either as absolute constraints (He et al, 1992) or weighted constraints. If the constraints are weighted, the option used here, they can be easily added to the least-squares normal equations by the summation of normals method. In our implementation, each constraint can be individually weighted by specifying a standard deviation. In practice, however, they are weighted as groups. A single standard deviation is defined for the group of base vector stability constraints. Likewise, a standard deviation is defined for the group of relative angle stability constraints.

The weighted constraints approach has the advantage of flexibility. The constraints can be effectively turned on or off. That is, they can be added if doing so is deemed to be appropriate: when the ROPs are indeed stable. Specifying very low group standard deviations allows the constraints to effectively become absolute constraints. In a complementary way, the ROP stability can be assessed using the residuals of the weighted constraints. Sensor stability is an important issue and many efforts have been devoted to the subject (e.g. Shortis et al, 20011; Habib et al, 2014). Furthermore, if there is a large sample of added constraints, then they, along with other observable quantities, can be appropriately weighted by analysing the estimated variance components.

\section{Self-Calibrating Bundle Adjustment}

The self-calibrating bundle adjustment problem is formulated in terms of a constrained Gauss-Markov model. The general form of the linearized image coordinate observations is partitioned into three parameter groups

$$
\mathbf{A}_{\mathrm{e}} \hat{\boldsymbol{\delta}}_{\mathrm{e}}+\mathbf{A}_{\mathrm{i}} \hat{\boldsymbol{\delta}}_{\mathrm{i}}+\mathbf{A}_{\mathrm{o}} \hat{\boldsymbol{\delta}}_{\mathrm{o}}+\mathbf{w}_{\mathrm{p}}=\hat{\mathbf{v}}_{\mathrm{p}}
$$

where $\mathbf{A}_{\mathrm{e}}, \mathbf{A}_{\mathrm{i}}$ and $\mathbf{A}_{\mathrm{o}}$ are the design matrices for the EOPs, the IOPs and the object point coordinates, respectively; $\delta_{\mathrm{e}}, \delta_{\mathrm{i}}$ and $\boldsymbol{\delta}_{\mathrm{o}}$ represent the correction vectors to the initial values for these three groups of parameters; $\mathbf{w}_{\mathrm{p}}$ is the misclosure vector; and $\mathbf{v}_{\mathrm{p}}$ is the vector of residuals. The associated weight matrix for this group of observations is demoted as $\mathbf{P}_{\mathrm{p}}$. The linearized ROP stability constraints are given by

$$
\mathbf{A}_{c} \hat{\boldsymbol{\delta}}_{\mathrm{e}}+\mathbf{w}_{\mathrm{c}}=\hat{\mathbf{v}}_{c}
$$

where $\mathbf{A}_{\mathrm{c}}, \mathbf{w}_{\mathrm{c}}$ and $\mathbf{v}_{\mathrm{c}}$ are the design matrix, misclosure vector and residual vector, respectively. The weight matrix for the constraints is demoted as $\mathbf{P}_{\mathrm{c}}$. The network datum 
is defined by inner constraints imposed on the object points

$$
\mathbf{G}_{\mathrm{o}}^{\mathrm{T}} \hat{\boldsymbol{\delta}}_{\mathrm{o}}=\mathbf{0}
$$

where $\mathbf{G}_{0}$ is the design matrix. All EOPs, IOPs and object points are simultaneously estimated in a multi-station, self-calibrating bundle adjustment of Equations 13-16 using established procedures (Luhmann et al, 2011).

\section{EXPERIMENTS}

\section{Calibration Facility}

Three datasets were captured with the Ladybug5 system in a dedicated indoor calibration laboratory at the University of Calgary: two for calibration and one for accuracy assessment. The laboratory is a temperature- and lighting-controlled environment and has nominal dimensions of $11 \mathrm{~m}$ x $11 \mathrm{~m}$ x $4 \mathrm{~m}$. Some 500 Bubble-X targets are mounted on the floor, the ceiling and the walls of the lab (Fig. 2). Bubble-X is an extruded plastic with two outer skin layers and an internal lattice structure that makes it considerably stiffer than paper or cardboard printed targets. It is a light-weight material and is not influenced by humidity. The target pattern comprises a white circle on a black background that is printed directly on the matte finish surface. Several different target sizes exist in the room. Those with $125 \mathrm{~mm}$ radius were used for this work. Some temporary paper targets were also mounted on the floor to densify the network.

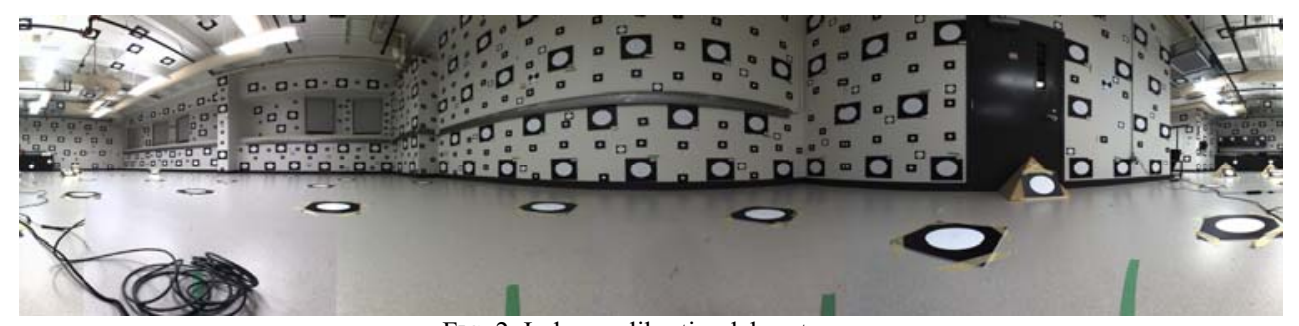

FIG. 2. Indoor calibration laboratory.

\section{Networks}

For calibration dataset 1, images were captured from three nominal locations with the Ladybug5 upright (Fig. 3). At each location, nine sets of images were captured. The camera system was rotated about its vertical axis by approximately $40^{\circ}$ between acquisitions so that the entire room was imaged by each of the horizontal cameras. This also provided convergent imaging geometry for the horizontal cameras and roll diversity for the vertical camera. Scale variation for the vertical camera imagery was provided by depth variation in the target field itself: targets positioned at different heights on the unfinished ceiling and on the walls were observed. Unfortunately, however, this network design did not include roll angle diversity for the horizontal cameras. Note that many targets on the east side of the room (Fig. 3) were omitted due to very narrow intersection 
angle geometry. Both these issues were rectified in calibration dataset 2 .
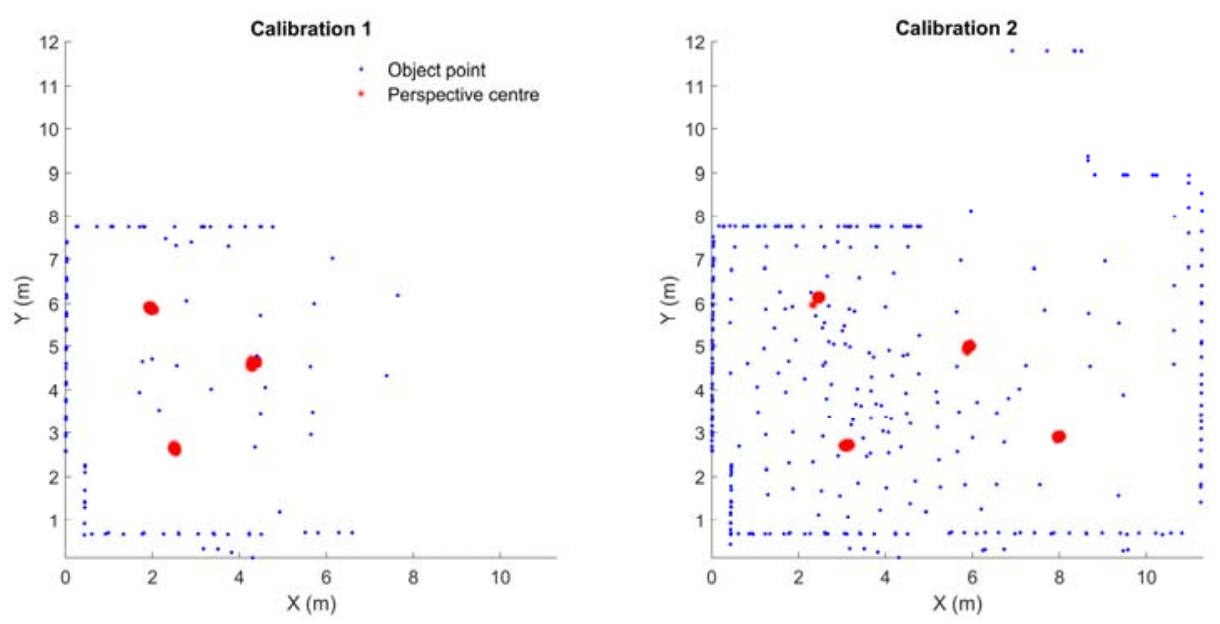

FIG. 3. Calibration networks.

Following a ten-month period during which the Ladybug5 was deployed on vehicleborne mobile mapping system and used for many data collection missions, calibration dataset 2 was captured with an improved network design (Fig. 3). This also provided an opportunity to quantify any changes in camera system geometry due to usage. The calibration network was densified with additional temporary, paper targets affixed to the floor in order to provide a more uniform distribution of observed points in the image plane. The network comprised four nominal camera locations. Three were approximately at the same positions as in calibration 1 while the fourth was closer to the east wall in so those targets could be imaged with improved intersection geometry. Some highly tilted images were also incorporated in the network. The Ladybug5 system was mounted on a monopod and inclined up to $45^{\circ}$ in two orthogonal directions at each nominal location. This provided the necessary roll diversity for the horizontal cameras. An Xsens MTI series inertial measurement unit was temporarily affixed to the camera system to observe the approximate EOP values needed for the target measurement algorithm and subsequent bundle adjustment.

\section{Accuracy Assessment}

The targets were independently surveyed to provide reference 3D coordinates for accuracy assessment. A single, high-resolution scan was acquired from the centre of the room with a Faro Focus 3D terrestrial laser scanner (TLS). A circular patch around each target was manually extracted from the point cloud. The centre coordinates were estimated using the method described by Lichti et al (2019b). Past experience with this instrument has demonstrated that millimetre-level 3D coordinate accuracy can be achieved in a similar environment with comparable dimensions (Lichti et al, 2019a).

Following the acquisition of calibration dataset 1, additional images were captured in the indoor calibration laboratory. These were used for accuracy assessment to quantify 
the effectiveness of the modelling approach proposed herein. The imagery was acquired from two exposure stations separated by $2.8 \mathrm{~m}$. These images were not used for the calibration but for independent accuracy assessment.

A total of 96 check points were observed in 10 horizontal images. Each image contained between 8 and 40 check points; all but two contained 20 or more. Almost all check points were observed in either two or three images. Two check points were observed in four images. The check points were estimated as tie points in the bundle adjustment. The ray intersection geometry of the tie points varied considerably, including both weakly- and strongly-determined points. Four control points in the corners of the network were fixed in the ensuing bundle adjustment to ensure that the estimated and reference coordinates from the TLS were in the same system. Check point coordinate differences were computed by subtracting the reference TLS coordinates from the photogrammetrically estimated values and the rmse $_{X Y Z}$ (Remondino et al, 2017) computed. Several different adjustment cases were performed for the accuracy assessment. These are described in more detail in subsequent sections.

\section{Image Point Measurement}

Images were captured in JPEG format since this is the standard operating procedure when the Ladybug5 is deployed as part of a vehicular MMS. The RGB colour imagery was converted to greyscale imagery according to the CIE 1931 colour space model:

$$
\text { Grey }=0.2125 R+0.7154 G+0.0721 B
$$

Measurement of the 2D target centre coordinates was performed using the automated detection and labelling algorithm described in Jarron et al (2019b). The major steps of the algorithm can be summarized as follows. Adaptive local thresholding of the grey scale image is performed followed by connected components analysis to label each region in the resulting binary image. RANSAC-based ellipse fitting is performed to eliminate outliers, which is followed by a final least-squares ellipse fit. A number of diagnostic tests are made to eliminate non-target regions. Prior knowledge, such approximate camera height, is utilized for some of the EOPs while a range of candidate values is assumed for other EOPs. The known 3D target coordinates of the calibration room are projected into the image using the EOPs and nominal camera IOPs. The image point corresponding to each projected object point is selected according to the minimum distance. This process is accompanied by tests to reduce the occurrence of incorrect labelling.

\section{Relative Orientation Stability Constraint Weighting}

As mentioned, the weighted constraints approach offers great flexibility for the enforcement of ROP stability. Here, three enforcement schemes were investigated: no constraints; small variances; variances set by approximate iterative variance component estimation (VCE). The high weights of the second approach meant that the constraints were effectively absolute. The aim of the third approach was to determine the weights of each group of observables (image coordinates, base vector stability constraints and 
relative angle stability constraints) such that their relative contribution is approximately equivalent.

\section{EXPERIMENT RESULTS}

\section{Adjustment Metadata}

Table I provides a high-level summary of the two calibration datasets. Overall, calibration 2 comprised fewer images (and, hence, fewer ROP stability constraints) but had more object points, image point observations and degrees-of-freedom. As can be seen in Fig. 4, the observations of both datasets cover nearly the entire image plane, respectively reaching $94 \%$ and $91 \%$ of the maximum radial distance of 1596 pixels. The distribution of observations was improved in the second dataset thanks to the additional targets on the floor.

TABLE I. Calibration dataset metadata.

\begin{tabular}{lcc}
\hline & Calibration 1 & Calibration 2 \\
\hline \# images & 262 & 206 \\
\# object points & 119 & 362 \\
\# observed image points & 6314 & 9742 \\
\# ROP stability constraint sets & 627 & 402 \\
Total degrees-of-freedom & 11903 & 17913 \\
Maximum observed radial distance (pix) & 1495 & 1456 \\
\hline
\end{tabular}

\section{Interior Orientation Parameter Estimates}

The estimates and the precision of the basic interior orientation parameters from the two calibration datasets are tabulated in Table II. Note that these results are from the case of very small variances for the ROP stability constraints. The horizontal cameras' $x_{p}$ precision is about $25 \%$ lower than that of $y_{p}$ for calibration 1 due to the aforementioned lack of roll diversity. The inclusion of highly tilted imagery in the second calibration dataset yielded the desired homogeneous precision. The principal point precision for the vertical camera was homogeneous for both calibrations due to the network design.

Principal distance precision among the horizontal cameras was homogeneous in both datasets, with first being slightly higher. In both cases, the vertical camera's precision is lower. In the first case, this is due to the lack of convergent imaging while in the second case it was due to the lower number of observations appearing in the vertical camera imagery. The scale variation did successfully de-correlate the PD from the EOPs in the first calibration as the largest correlation coefficient with the perspective centre position was 0.2 . In the second calibration, the introduction of highly-convergent vertical camera images meant that many targets on the unfinished ceiling were partially or completely occluded by ductwork, electrical conduit, water pipes and light fixtures. As a result, the precision is lower. But, de-correlation from the EOPs was also achieved as the largest correlation was 0.15 . 

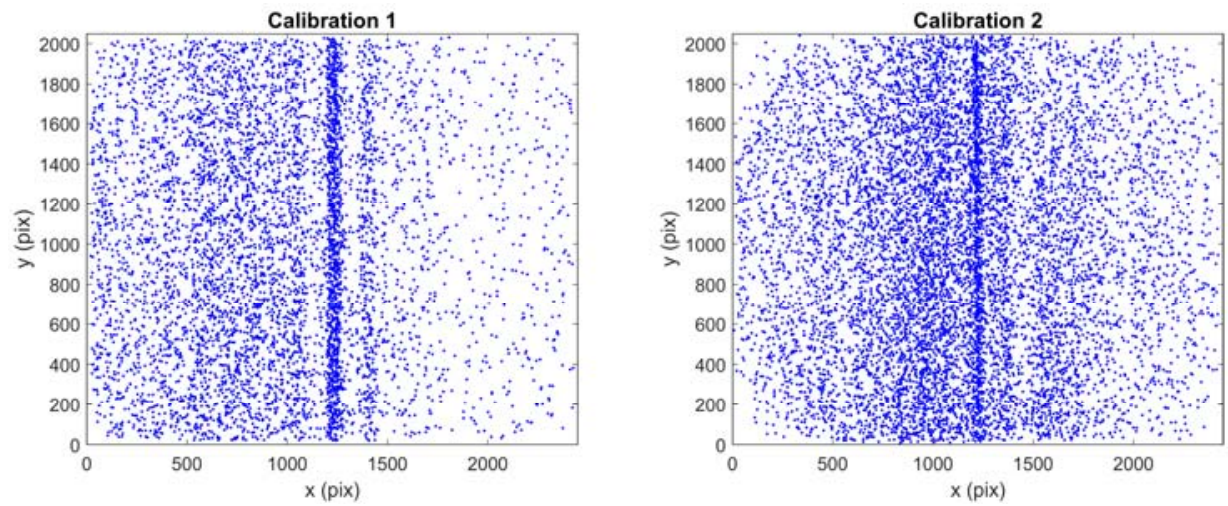

FIG. 4. Image point distribution for the calibration datasets (all images, all cameras).

Analysing Table II reveals there are differences in the basic IOPs of the two calibration datasets. All are 0.8 pix or less except for $y_{p}$ and the principal distance of the vertical camera, about 1.4 pix and 2.1 pix, respectively. These can be explained by the already-described network differences. Under the reasonable assumption that the parameter estimates from the two datasets are uncorrelated, statistical testing was performed to assess the significance of these differences. Seven parameters were identified as significantly different at the $95 \%$ confidence level. Noting, however, that the linear equivalent of 0.8 pix in object space is approximately $6 \mathrm{~mm}$ in object space, which is smaller than the mean object point precision from the check point analysis (see next section), these results suggest that the remaining parameters of the interior geometry were stable over the time between calibrations.

TABLE II. Estimated interior orientation parameters from the two calibration datasets. Pairs of parameter estimates that are significantly different are shown in bold.

\begin{tabular}{cccccccc}
\hline & & \multicolumn{7}{c}{ Horizontal Cameras } & Vertical \\
\cline { 3 - 8 } IOP & Calibration & 0 & 1 & 2 & 3 & 4 & 5 \\
\hline$x_{p}$ & 1 & $\mathbf{1 2 0 8 . 6 1}$ & 1205.25 & 1235.22 & 1226.66 & 1234.21 & $\mathbf{1 2 1 8 . 0 0}$ \\
(pix) & 2 & $\mathbf{1 2 0 9 . 0 8}$ & 1205.49 & 1234.85 & 1226.73 & 1234.33 & $\mathbf{1 2 1 8 . 3 6}$ \\
$\sigma$ & 1 & 0.16 & 0.16 & 0.15 & 0.15 & 0.15 & 0.07 \\
$($ pix) & 2 & 0.11 & 0.12 & 0.12 & 0.11 & 0.11 & 0.14 \\
$y_{p}$ & 1 & $\mathbf{1 0 1 2 . 7 4}$ & $\mathbf{1 0 2 0 . 3 7}$ & 1016.62 & 1000.33 & 1021.52 & $\mathbf{1 0 1 6 . 4 7}$ \\
$($ pix) & 2 & $\mathbf{1 0 1 1 . 9 5}$ & $\mathbf{1 0 1 9 . 9 9}$ & 1016.57 & 1000.16 & 1021.81 & $\mathbf{1 0 1 7 . 8 5}$ \\
$\sigma$ & 1 & 0.12 & 0.11 & 0.11 & 0.11 & 0.12 & 0.06 \\
$($ pix) & 2 & 0.12 & 0.13 & 0.13 & 0.12 & 0.12 & 0.13 \\
$c$ & 1 & 1234.91 & 1235.86 & $\mathbf{1 2 3 9 . 5 4}$ & 1240.38 & 1239.51 & $\mathbf{1 2 3 9 . 4 6}$ \\
$($ pix $)$ & 2 & 1235.25 & 1235.82 & $\mathbf{1 2 3 8 . 9 5}$ & 1240.11 & 1239.08 & $\mathbf{1 2 3 7 . 3 4}$ \\
$\sigma$ & 1 & 0.18 & 0.17 & 0.17 & 0.18 & 0.19 & 0.35 \\
$($ pix $)$ & 2 & 0.22 & 0.22 & 0.21 & 0.21 & 0.22 & 0.59 \\
\hline
\end{tabular}

\section{Radial Lens Distortion}

The development of the radial lens distortion model is demonstrated with calibration dataset 1 without enforcement of the relative orientation constraints. If the constraints are 
heavily weighted, their addition to the bundle adjustment can lead to inflation of the image point. Thus, they were omitted so as to prevent possible biases in this model development exercise.

TABLE III. Model fit as a function of increasing the number of radial lens distortion terms for calibration 1.

\begin{tabular}{lcc}
\hline Radial lens distortion model terms & $r m s_{x y}($ pix $)$ & \% improvement \\
\hline$k_{1}, k_{2}$ & 4.94 & - \\
$k_{1}, k_{2}, k_{3}$ & 1.21 & 73 \\
$k_{1}, k_{2}, k_{3}, k_{4}$ & 0.45 & 90 \\
$k_{1}, k_{2}, k_{3}, k_{4}, k_{5}$ & 0.38 & 92 \\
\hline
\end{tabular}

The model fit following sequential addition of lens distortion terms is reported in Table III. The $r m s_{x y}$ values are pooled estimates from all six cameras. The "base" model comprises the first two terms, $k_{1}$ and $k_{2}$. The adjustment including only $k_{1}$ did not converge due to the extreme barrel distortion of the Ladybug5 system. The $r m s_{x y}$ estimates show a clear but gradually diminishing improvement as a result of including each successive radial lens distortion term. The final, five-term model exhibits an order of magnitude from improvement over the initial, two-term model. Analysis of the AIC revealed that the final model was indeed superior rot he others. Note that the final $r m s_{x y}$ is lower than that reported in Table I due to omission of the ROP stability constraints.
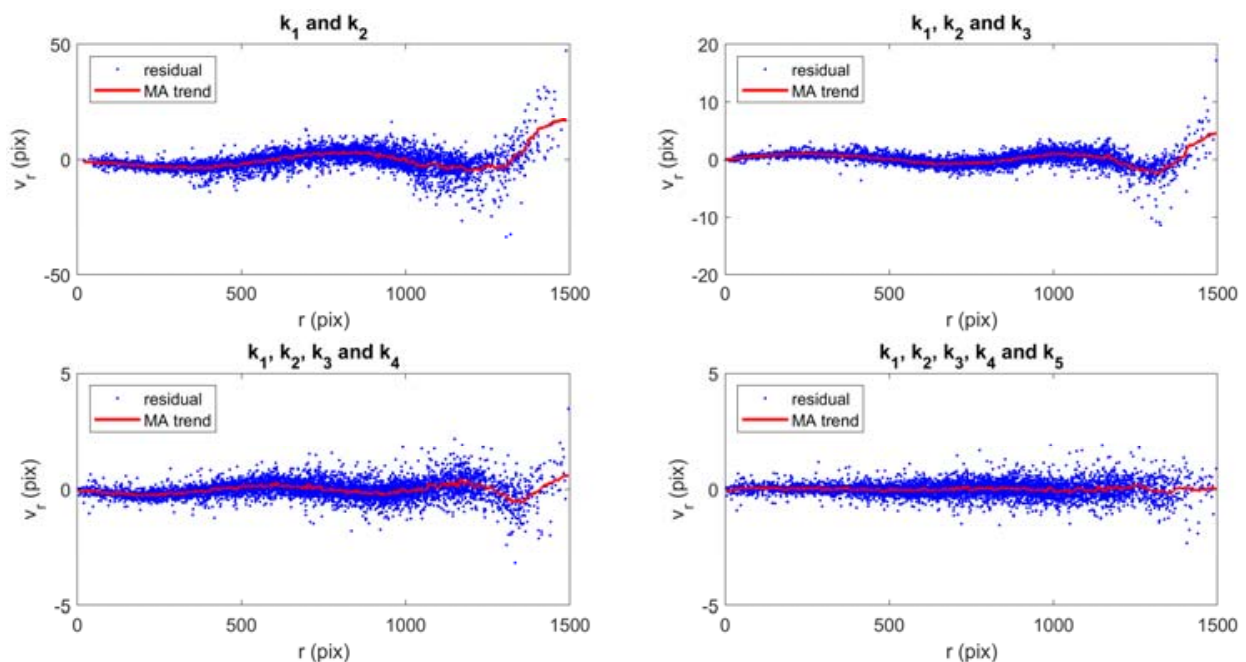

FIG. 5. Radial component of the image point residuals $\left(v_{r}\right)$ and moving average (MA) trend for calibration 1 .

Graphical analyses were used to support the model development. The scatter plots in Fig. 5 represent the superimposition of the radial component for all residuals $\left(v_{r}\right)$ from all six cameras. The moving average (MA) trends show the presence of un-modelled systematic distortions, except in the final model. Note that un-modelled trends do not reflect the exact behaviour of the missing term(s) but the residual errors from the other terms trying to compensate for the missing terms, hence the multiple concavities in the 
first three figures. The final case with all 5 terms shows a flat MA trend, indicating that all systematic effects have been modelled up to the maximum observed radial distance of 1495 pix.

TABLE IV. Radial lens distortion term significance for calibration 1.

\begin{tabular}{lcc}
\hline Radial lens distortion model terms & Total number of terms & Number of significant terms \\
\hline$k_{1}, k_{2}$ & 12 & 12 \\
$k_{1}, k_{2}, k_{3}$ & 18 & 18 \\
$k_{1}, k_{2}, k_{3}, k_{4}$ & 24 & 24 \\
$k_{1}, k_{2}, k_{3}, k_{4}, k_{5}$ & 30 & 29 \\
\hline
\end{tabular}

The statistical significance of each radial lens distortion term was evaluated. In all model cases, all terms are significant at the $95 \%$ confidence level with only one exception: $k_{2}$ for the vertical-looking camera with the full model (Table IV). In this instance, the test value was 1.92, so it was in fact only marginally insignificant. Statistical testing for the second calibration dataset revealed that all 30 terms of the full model were significant. These outcomes, should, however, be tempered by the fact that high correlations - that are ignored in the individual significance testing - exist among the radial lens distortion coefficients. An example of the correlation coefficients for one camera is given in Table V. Some are quite large. Importantly, though, there were no high correlations with any of the EOPs; the largest coefficient was 0.46 .

TABLE V. Sample correlation coefficient matrix for radial lens distortion coefficients.

\begin{tabular}{cccccc}
\hline & $k_{1}$ & $k_{2}$ & $k_{3}$ & $k_{4}$ & $k_{5}$ \\
\hline$k_{1}$ & 1 & & & & \\
$k_{2}$ & -0.974 & 1 & & & \\
$k_{3}$ & 0.931 & -0.988 & 1 & 1 & \\
$k_{4}$ & -0.885 & 0.962 & -0.993 & -0.995 & 1 \\
$k_{5}$ & 0.843 & 0.933 & 0.976 & & \\
\hline
\end{tabular}

The best measure of the effectiveness of the radial lens distortion model is the independent accuracy assessment with check points (Table VI). Similarly to the results in Table III, the addition of each model term results in a significant, but diminishing, improvement. A bias of up to $1 \mathrm{~cm}$ exists in the first two models and the third case does not meet precision expectations. The five-term model is the only one for which the rmse $e_{X Y Z}$ met the expected accuracy of $13 \mathrm{~mm}$, the mean tie point coordinate precision. Thus, despite the high correlations between terms, the inclusion of five radial lens distortion terms improves within a laboratory setting.

TABLE VI. Accuracy assessment as a function of the number of radial lens distortion terms for calibration 1.

\begin{tabular}{lccc}
\hline Radial lens distortion model terms & Mean difference $(\mathrm{mm})$ & rmse $_{X Y Z}(\mathrm{~mm})$ & \% improvement \\
\hline$k_{1}, k_{2}$ & 8 & 152 & - \\
$k_{1}, k_{2}, k_{3}$ & 10 & 36 & 76 \\
$k_{1}, k_{2}, k_{3}, k_{4}$ & 3 & 18 & 88 \\
$k_{1}, k_{2}, k_{3}, k_{4}, k_{5}$ & 2 & 10 & 93 \\
\hline
\end{tabular}


Both decentring distortion parameters were statistically significant for both calibration datasets. However, the decentring effect is considerably smaller than that of the radial lens distortion. The decentring profile reaches only 2.1 pix at the maximum radial distance. Therefore, decentring distortion was not analysed further.

\section{Model Fit and Relative Orientation Parameters}

Table VII reports the rms for the image points, the base vector components and the relative angles. The ROPs were derived from the estimated EOPs following adjustments with all three enforcement schemes. Regardless of the weighting scheme used, the quality of the fit of the observations to the sensor model is slightly lower in the second dataset. For both datasets, the imposition of the small-variance ROP stability constraints had the effect of inflating the $r m s_{x y}$. However, the constraints were clearly effective in enforcing ROP stability as the rms values from a couple millimetres and a couple degrees to insignificance. The constraint enforcement case with VCE-derived weights had less impact in terms of inflating the image point coordinate residuals. ROP stability was enforced to the level of about one-quarter of a millimetre for base vector components and less than one-half of an arc minute for the relative angles.

TABLE VII. Derived relative orientation parameter statistics. Note the different units for the $r s_{\text {angle }}$ column.

\begin{tabular}{ccccc}
\hline Calibration & ROP stability enforcement & $r m s_{x y}(p i x)$ & $r m s_{\text {base }}(\mathrm{mm})$ & $r m s_{\text {angle }}\left({ }^{\prime \prime \prime}\right)$ \\
\hline 1 & None & 0.38 & 2.48 & $2.16^{\circ}$ \\
1 & Small variance & 0.48 & 0.002 & $0.04^{\prime \prime}$ \\
1 & VCE & 0.42 & 0.28 & $26^{\prime \prime}$ \\
2 & None & 0.61 & 1.93 & $3.76^{\circ}$ \\
2 & Small variance & 0.66 & 0.000 & $0.04^{\prime \prime}$ \\
2 & VCE & 0.62 & 0.24 & $23^{\prime \prime}$ \\
\hline
\end{tabular}

Differences between the ROPs of the two calibrations were computed for the small variance enforcement scheme. The statistics are presented in Table VIII. The results suggest that even after repeated use, the relative geometry of the Ladybug5 system is stable at the 1-2 millimetre level.

TABLE VIII. Statistics of the differences between the derived relative orientation parameters from calibrations 1 and 2.

\begin{tabular}{lccc}
\hline & Base vector components $(\mathrm{mm})$ & Relative angles (") & Linear equivalent at $10 \mathrm{~m}(\mathrm{~mm})$ \\
\hline Mean & -0.5 & -25 & -1.2 \\
Maximum & 1.9 & 79 & 3.8 \\
$r m s$ & 0.8 & 40 & 1.9 \\
\hline
\end{tabular}

\section{Accuracy Assessment-ROP Stability Constraints}

As mentioned, several adjustments were performed for the accuracy assessment. Pre-determined IOPs were implemented as constants and pre-determined ROPs, 
implemented as weighted constraints (Equations 7, 10,11 and 12) with small variances so as to effectively make them absolute constraints. IOP and ROP estimates from all three weighting schemes were tested. Each adjustment comprised 198 degrees-of-freedom. Jarron et al (2019a) provide an initial report on differences between estimated and factory calibration parameters. Since very large differences between radial lens distortion profiles beyond $\mathrm{r}=1000$ pix are reported, the factory-determined values were not used. Several combinations of factory parameters and parameters determined by our methodology were tested and are summarized in Table IX.

TABLE IX. Accuracy assessment statistics. All weighting schemes are small variance unless otherwise indicated

\begin{tabular}{|c|c|c|c|c|}
\hline & $\begin{array}{c}\text { rmse }_{X Y Z} \\
(\mathrm{~mm})\end{array}$ & $\begin{array}{c}\% \\
\text { improvement }\end{array}$ & $\begin{array}{c}3 D \text { coordinate } \\
\text { difference range }(\mathrm{mm})\end{array}$ & $\begin{array}{c}\% \\
\text { improvement }\end{array}$ \\
\hline $\begin{array}{l}\text { Factory ROPs, } x_{p}, y_{p} \text { and } c \text {, our } \\
\text { lens distortions (calibration } 1 \text { ) }\end{array}$ & 133 & - & 1391 & - \\
\hline $\begin{array}{l}\text { Factory ROPs, our IOPs } \\
\text { (calibration } 1 \text { ) }\end{array}$ & 76 & 43 & 675 & 52 \\
\hline $\begin{array}{l}\text { Our IOPS, no ROPs } \\
\text { (calibration 1) }\end{array}$ & 10 & 92 & 95 & 93 \\
\hline $\begin{array}{l}\text { Our IOPs and ROPs } \\
\text { (calibration 1) }\end{array}$ & 9 & 93 & 68 & 95 \\
\hline $\begin{array}{l}\text { Our IOPs and ROPs } \\
\text { (calibration 2) }\end{array}$ & 10 & 92 & 95 & 93 \\
\hline $\begin{array}{l}\text { Our IOPs and ROPs } \\
\text { (calibration } 1, \mathrm{VCE} \text { ) }\end{array}$ & 7 & 95 & 49 & 96 \\
\hline $\begin{array}{l}\text { Our IOPs and ROPs } \\
\text { (calibration 2, VCE) }\end{array}$ & 10 & 92 & 94 & 93 \\
\hline
\end{tabular}

The first case using factory ROPs, some factory IOPs and our lens distortion coefficients shows decimetre-level agreement of photogrammetrically determined and scanned coordinates, but the range in coordinate differences is greater than metre-level. The large differences exist at weakly determined points that nominally lie on the baseline between camera stations (Fig. 6). Utilizing all our IOPs considerably improves the results by $43 \%$ in $r m s_{X Y Z}$ and $52 \%$ in terms of the $3 \mathrm{D}$ coordinate difference range. The $3 \mathrm{D}$ range is the diagonal of the rectangular prism spanned by the range of coordinate differences in each dimension. Centimetre-level accuracy is achieved with IOPs (without known ROPs enforced). Adding the known ROPs as constraints does not improve accuracy in terms of $r m s_{X Y Z}$, but does reduce the range of coordinate differences. The improvement is gained is in the outliers that occur at the narrow intersection angles (Fig. 6). Without the ROP constraints, the differences in any one coordinate were up to $4 \mathrm{~cm}$. With the constraints, differences were up to $3 \mathrm{~cm}$. Using the IOPs and ROPs from the second calibration produces similar results, though the range of differences is degraded slightly due to the changes in parameters reported in Tables II and VIII.

For all cases discussed in the preceding paragraph, the IOPs and ROPs were derived from the small-variance weighting scheme. Use of the parameters from the VCE weighting scheme (final two rows of Table IX) produced comparable accuracy to that achieved with the small variance parameters. In fact, the calibration 1 results are slightly superior in terms of both measures. The slightly less accurate results using the calibration 2 parameters are in line with the findings above. 

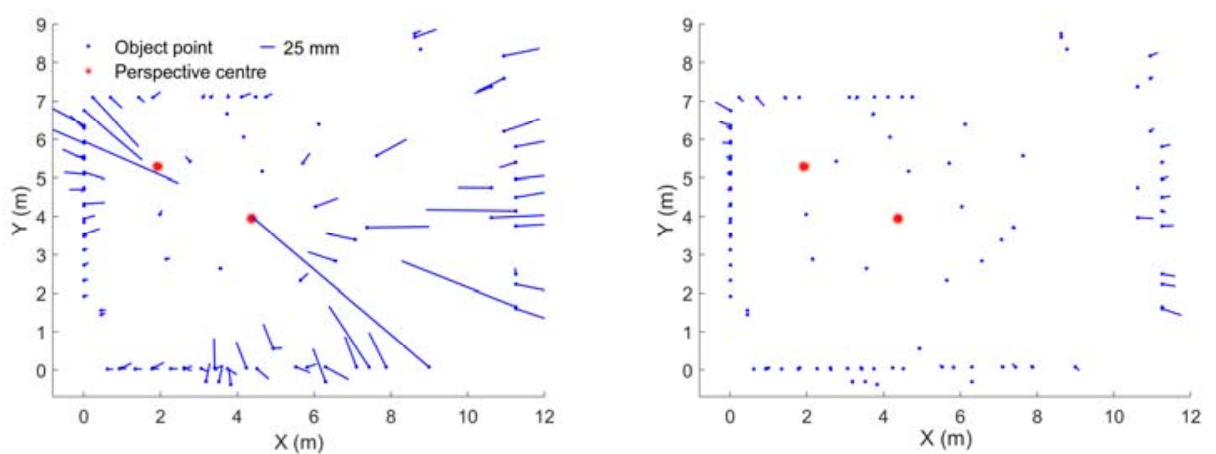

FIG. 6. Check point coordinate differences. Left: with factory ROPs, $x_{p}, y_{p}$ and $c$, our lens distortions (calibration 1). Right: with our IOPs and ROPs (calibration 1).

\section{ROP Stability Constraints—Parameter Correlations}

The ROP stability constraints impose functional dependencies between EOPs. Although their effectiveness has been demonstrated, the question of whether the solution is degraded by the small- variance weighting. Therefore, an analysis of correlations among EOPs and with IOPs has been undertaken. Table X presents a summary of large correlation coefficients, defined as having magnitude greater than 0.9 , for all three weighting schemes for both datasets.

Using the unconstrained case as a benchmark, imposing the small-variance stability constraints increases the number of high EOP-EOP correlations by nearly two orders of magnitude for both calibrations. The high correlations in the unconstrained case are due to local weaknesses in network geometry that are exacerbated by the constraints. Interestingly, the VCE weighting actually reduces, albeit very slightly, the number of high EOP-EOP correlations, so there is no adverse effect caused by their introduction.

The low number of high EOP-IOP correlations in the unconstrained case demonstrates the success of the network design to reduce projective compensation. The use of the small-variance constraints introduces several hundred high correlations. The VCE weighting does introduce some correlations, but the number is an order of magnitude smaller.

TABLE X. Number of correlation coefficients with magnitude greater than 0.9.

\begin{tabular}{cccc}
\hline Calibration & ROP stability enforcement & EOP-EOP & EOP-IOP \\
\hline 1 & None & 226 & 0 \\
1 & Small variance & 20168 & 754 \\
1 & VCE & 218 & 36 \\
2 & None & 184 & 26 \\
2 & Small variance & 8957 & 512 \\
2 & VCE & 182 & 52 \\
\hline
\end{tabular}

It should be borne in mind that the total number of off-diagonal elements in the parameter correlation matrices is large: 3942210 and 5671542 for calibrations 1 and 2, 
respectively. Thus, the percentage of large correlations in the small variance case is less than $1 \%$. However, the introduction of these dependencies may be the cause of the slightly worse results for the small-variance accuracy assessment for calibration 1 in comparison with the VCE results. In terms of good practice, the VCE enforcement approach is recommended since it avoids the introduction of adverse correlations.

\section{CONCLUSIONS}

An approach for the geometric modelling and calibration of a multi-camera, spherical imaging system, the Ladybug5, has been presented. Results from multiple calibration datasets were presented to demonstrate the effectiveness of the methodology. Image coordinate observations agreed with the model at the sub-pixel level. Centimetrelevel 3D reconstruction accuracy in object space was achieved using the parameters determined with the proposed approach. Accuracy was improved by $95 \%$ was over what could be achieved using the manufacturer's interior orientation and relative orientation parameters. The severe barrel distortion caused by the system's wide-angle lenses necessitated the adoption of a five-term radial lens distortion model. Although this is unconventional and the distortion coefficients are inherently very highly correlated, it was found to be successful. Inclusion of five radial lens distortion terms led to a $92 \%$ improvement in model fit in terms of residual rms and a 93\% improvement in 3D object space accuracy. The relative position and orientation of the system's six cameras were effectively modelled with relative orientation stability constraints implemented as weighed constraints. Analysis of the system calibration after a ten-month period of usage revealed sub-pixel stability of most parameters of the interior orientation and millimetrelevel stability of the relative orientation parameters.

Two different approaches to weighting the ROP stability constraints were investigated. It may be tempting to enforce the constraints rigidly with very small variances (high) weights to accurately model the assumed mechanical stability among the individual sensors of a multi-camera system. However, the approach of determining the stability constraint weights using VCE has been demonstrated to be superior for the calibration of the Ladybug5 system. The relaxed weighting achieved comparable (or slightly better) accuracy, did not introduce adverse parameter correlations and resulted in only minor inflation of the image point coordinate residuals.

A number of possibilities for future work exist. Perhaps paramount among these is the possibility to model wide-angle lenses like those of the Ladybug5 with a fisheye lens model. A preliminary investigation of this approach yielded promising results (Jarron et al., 2019). The concept is the fisheye model compensates for the majority of the barrel distortion, leaving only a residual radial lens distortion signal that should require fewer coefficients. Although the high correlations between coefficients did not degrade the results in this study, it would be advantageous to utilize a model free from strong functional dependencies as a general principle. Scope exists for future investigation into the use of the VCE weighting for the ROP stability constraints applied to other multicamera systems and the exact correlation mechanisms. 


\section{ACKNOWLEDGEMENTS}

Funding for this research was provided by the Natural Sciences and Engineering Research Council of Canada and McElhanney Ltd.

\section{REFERENCES}

Agisoft LLC, 2018. Agisoft PhotoScan User Manual Professional Edition, Version 1.4, 121 pp.

Granshaw, S. I., 2016. Photogrammetric terminology: third edition. Photogrammetric Record, 31(154): 210251.

AKAIKE, H., 1974. Information theory and an extension of the maximum likelihood principle. IEEE Transactions on Automatic Control, 19(1): 716-723.

Campos, M.B, Tommaselli, A.M.G., Marcato Junior, J. and HonkavaAra, E., 2018. Geometric model and assessment of a dual-fisheye imaging system. Photogrammetric Record, 33(162): 245-263.

Bosch, J., Gracias, N., RidAO, P. and RIBAS D., 2015 Omnidirectional Underwater Camera Design and Calibration. Sensors, 15: 6033-6065

CUI, T., JI, S., SHAN, J., GONG, J. and LiU, K., 2017. Line-Based Registration of Panoramic Images and LiDAR Point Clouds for Mobile Mapping. Sensors, 17: 70.

Detchev, I., HabiB, A., MAZAherI, M. and Lichti, D., 2018. Practical in-situ implementation of a multicamera multi-system calibration. Journal of Sensors, 2018: 1-12.

HABIB, A., Kersting, A.P., BANG, K.-I. and RAU, J., 2011. A novel single-step procedure for the calibration of the mounting parameters of a multi-camera terrestrial mobile mapping system. Archives of Photogrammetry, Cartography and Remote Sensing, 22: 173-185.

Habib, A. Detchev, I. and KwAK, E., 2014. Stability Analysis for a Multi-Camera Photogrammetric System. Sensors, 14: 15084-15112.

He, G., NovaK, K. and FenG, W., 1992. On the integrated calibration of a digital stereo vision system. International Archives of the Photogrammetry, Remote Sensing and Spatial Information Sciences, XXIX(B5): 139-145.

Jarron, D., Lichti, D., Shahbazi, M., and Radovanovic, R., 2019a. Multi-Camera Panoramic Imaging System Calibration. In: Proceedings of the $11^{\text {th }}$ International Conference on Mobile Mapping Technology. Shenzhen, China, 6-8 May. 70-77.

Jarron, D., Shahbazi, M., Lichti, D., and Radovanovic, R., 2019b. Automatic detection and labelling of photogrammetric control points in a calibration test field. International Archives of Photogrammetry, Remote Sensing and Spatial Information Sciences, XLII-2/W13: 1673-1680.

Jarron, D., Shahbazi, M., Lichti, D., and RAdOVAnovic, R., 2019c. Modelling wide-angle lens cameras for metrology and mapping applications. ISPRS Annals of Photogrammetry, Remote Sensing and Spatial Information Sciences, IV-2/W7: 79-86.

KHORAMSHAHI, E., and HONKAVAARA, E., 2018. Modelling and automated calibration of a general multi-projective camera. Photogrammetric Record, 33(161): 86-112.

KING, B.A., 1995. Bundle adjustment of constrained stereopairs-mathematical models. Geomatics Research Australasia, 63: 67-92.

Lerma, J.L. Navarro, S., Cabrelles, M, and Segui, A.E., 2010. Camera calibration with baseline distance constraints. Photogrammetric Record, 25(130): 140-158.

Lichti, D.D., Sharma, G.B., Kuntze, G., Mund, B., Beveridge, J.E., Ronsky, J.L., 2015. Rigorous Geometric Self-Calibrating Bundle Adjustment for a Dual Fluoroscopic Imaging System. IEEE Transactions on Medical Imaging, 34(2): 589-598.

Lichti, D.D., GlenNie, C.L., Jahraus, A. and HartZell, P., 2019a. New approach for low-cost TLS target measurement. ASCE Journal of Surveying Engineering, 145(3): 04019008-1 - 04019008-12.

Lichti, D.D., Glennie, C.L., Al-Durgham, K., Jahraus, A. and Steward, J., 2019b. Explanation for the seam line discontinuity in terrestrial laser scanner point clouds. ISPRS Journal of Photogrammetry and Remote Sensing. 154, 59-69.

Lichti, D.D., JARRON, D., ShahbaZi, M., Helmholz, P. and Radovanovic, R., 2019c. Investigation into the behaviour and modelling of chromatic aberrations in non-metric digital cameras. International Archives of the Photogrammetry, Remote Sensing and Spatial Information Sciences, XLII-2/W18: 99-106.

PнотометRIX, 2016. CameraCalibrator User Manual Version 2.0. $47 \mathrm{pp}$.

Luhmann, T., Robson, S., Kyle, S. and Boehm, J., 2013. Close-Range Photogrammetry and 3D Imaging, $2^{\text {nd }}$ edition. De Gruyter. Berlin, 702 pp. 
LICHTI et al. Geometric modelling and calibration a spherical camera imaging system

RAU, J.Y., SU, B.W., HSIAO, K.W. and JHAN, J.P., 2016. Systematic calibration for a backpacked spherical photogrammetry imaging system. International Archives of the Photogrammetry, Remote Sensing and Spatial Information Sciences, XLI-B1: 695-702.

Remondino, F., Nocerino, E., TOSCHI, I. and MenNA, F. 2017. A critical review of automated photogrammetric processing of large datasets. International Archives of the Photogrammetry, Remote Sensing and Spatial Information Sciences, XLII-2/W5: 591-597.

SCHNEIDER, J. and FÖRSTNER, W., 2013. Bundle adjustment and system calibration with points at infinity for omnidirectional cameras. Institute of Geodesy and Geoinformation, University of Bonn, Germany. 22 pp.

Shortis, M.R., Ogleby, C.L., Robson, S., Karalis, E.M. and BeYER, H., 2001. Calibration modelling and stability testing for the Kodak DC200 series digital still camera. Videometrics and Optical Methods for 3D Shape Measurement, San Jose, CA, 22-23 January, 148-153.

SHORTIS, M., 2015. Calibration techniques for accurate measurements by underwater camera systems, Sensors, 15: 30810-30827.

Tommaselli, A.M.G., Galo, M., De Moraes, M.V.A., Marcato Junior, J., Caldeira, C.R.T. and Lopes, R.F., 2013. Generating virtual images from oblique frames. Remote Sensing, 5: 1875-1893.

WANG, Y., Chen, Q., ZHU, Q., LiU, L., Li, C. and Zheng, D., 2019. A survey of mobile laser scanning applications and key techniques over urban areas. Remote Sensing, 11: 1540.

\section{Résumé}

L'histoire de l'appariement d'images remonte à plus de cinquante ans, lorsque les premières ...

\section{Zusammenfassung}

Die digitale Bildzuordnung hat seit den ersten analogen Ansätzen für die automatisierte ...

\section{Resumen}

La correspondencia de imágenes tiene una historia de más de 50 años, desde los primeros ...

摘要

影像匹配技术在模拟摄影测量中首次应用开始，已经有50年的发展 ‥ 